\title{
Abdominal Pain from a Thoracic Epidural Abscess
}

\author{
Erle Chuen-Hian Lim, M Med (Int Med), Raymond C.S. Seet, MRCP \\ Division of Neurology, National University Hospital, Singapore, Singapore.
}

\begin{abstract}
Spinal epidural abscesses are difficult to diagnose and are associated with high morbidity and mortality. A 61-year-old Chinese woman fell and was admitted 5 days later with constant epigastric pain and constipation. Clinical examination was initially normal. Five days after admission, she developed urinary retention and mild lower limb weakness, progressing overnight to paraplegia. Clinical examination was consistent with a thoracic myelopathy. Neuroimaging showed a paracentral fluid collection compressing the thoracic cord at T8 level. Emergent neurosurgical intervention revealed an epidural abscess, which was drained. She responded to a 9-week course of antibiotics and recovered fully. Radicular pain from thoracic pathology can mimic intraabdominal pathology, and a high index of suspicion and prompt surgical intervention are essential to avoid the potentially devastating consequences of delayed recognition of epidural abscesses.
\end{abstract}

KEY WORDS: abdominal; pain; epidural abscess; thoracic. DOI: $10.1111 / \mathrm{j} .1525-1497.2005 .00259 . x$ J GEN INTERN MED 2006; 21:C8-C10.

A spinal epidural abscess (SEA) is a collection of pus, lying outside the dura mater, but within the spinal canal. Spinal epidural abscesses are rare, and are diagnosed in only 1 out of 10,000 admissions to hospital. ${ }^{1}$ Despite the difficulty in diagnosing SEA, ${ }^{2}$ it is important to do so early, as timely neurosurgical intervention can prevent progression to permanent devastating neurologic deficit. Rarely, SEAs involving the thoracic spine can present with abdominal pain, ${ }^{3,4}$ from involvement of the thoracic nerve roots. ${ }^{5}$ We describe a patient with a thoracic SEA, who presented initially with epigastric pain and constipation, and later developed urinary retention and paraplegia.

\section{CASE REPORT}

A 61-year-old Chinese female was admitted with 5 days' complaint of constipation associated with abdominal pain. Her medical history included uncomplicated hypertension and diabetes mellitus, for which she was on dietary control. Her abdominal pain, which was located over the epigastrium, was described to be noncolicky, constant, unrelated to meals, exacerbated by movement, and severe enough to interrupt her sleep at night. It did not radiate into the back, and was rated at 7 to 8 on a pain scale of 1 to 10 (10 equated with the pain from childbirth). There was no associated nausea or vomiting. She recalled having slipped and fallen 1 day prior to onset of symptoms. Despite having mild back pain, she denied motor or sensory complaints before or after the fall. She had no past history

The authors have no conflicts of interest to declare.

Address correspondence and reprint requests to Dr. Lim: National University Hospital, 5 Lower Kent Ridge Road, Singapore 119074, Singapore (e-mail: mdcelch@nus.edu.sg).

C8 of urinary tract infections. She had mild fever of $37.4{ }^{\circ} \mathrm{C}$, and the clinical examination, including tone, deep tendon reflexes, plantar response, motor power, and sensation to pinprick and proprioception, was normal.

Blood tests showed polymorphonuclear leukocytosis $\left(12.38 \times 10^{9}\right.$ leukocytes/L, range 3.40 to 9.20$)$, and elevation of both erythrocyte sedimentation rate $(98 \mathrm{~mm} / \mathrm{hr}$, range 5 to 15) and C-reactive protein ( $18.9 \mathrm{mg} / \mathrm{dL}$, range 0.0 to 1.0 ). Calcium levels, hepatic and cardiac biomarkers were normal. Serum electrolytes were likewise within normal limits, except for mild hyponatremia of $122 \mathrm{mmol} / \mathrm{L}$ (range 135 to 150), which normalized with judicious intravenous hydration. She was hyperglycemic, with serum glucose of $12.9 \mathrm{mmol} / \mathrm{L}$ (range 4.0 to 7.8 ), and glycated hemoglobin was measured at $8.1 \%$ (goal $<7 \%$ ). Urinalysis was normal. Serum amylase and lipase were not assayed. Abdominal radiograph, hepatobiliary sonogram, and endoscopy of the upper and lower gastrointestinal tracts were normal, as were electrocardiogram and chest radiograph. She remained distressed by the epigastric pain.

Five days after admission, she developed lower limb weakness, progressing over the course of the day to flaccid paraplegia. That same day, she developed urinary retention, which necessitated urinary catheterization. A neurology consultation was sought. Clinical examination was significant for absence of superficial abdominal reflexes and flaccid paraparesis of the lower limbs, with motor power of Medical Research Council (MRC) grade 2/5 (i.e., she was able to move them from side to side, but not overcome gravity). Pinprick and temperature sensory loss in the lower limbs were elicited, but she was unable to cooperate fully with the examination because of pain and emotional distress, and a definitive sensory level could not be determined. She had paravertebral muscle spasm, and thoracic vertebral tenderness. Examination of the upper extremities was within normal limits.

A provisional diagnosis of thoracic myelopathy was made, the hyporeflexia being attributed to spinal shock, i.e., temporary depression of all or most spinal reflex activity below the level of injury from acute spinal cord injury. ${ }^{6}$ Thoracic magnetic resonance imaging was obtained, showing cord edema from 6th to 8th thoracic (T6 to T8) levels and a left anterior paracentral compression of the spinal cord at T8. This was consistent with an epidural abscess or hematoma (Figs 1, A and $\mathrm{B}$ and $2, \mathrm{~A})$. In addition, there was a septated paravertebral abscess at T6 to T8 levels (Fig. 2, B).

Emergent laminectomy was performed from the 6th to 8th thoracic vertebrae, during which an epidural abscess was drained. Cultures of the purulent fluid grew Staphylococcus

Received for publication March 29, 2005

Accepted for publication August 10, 2005 

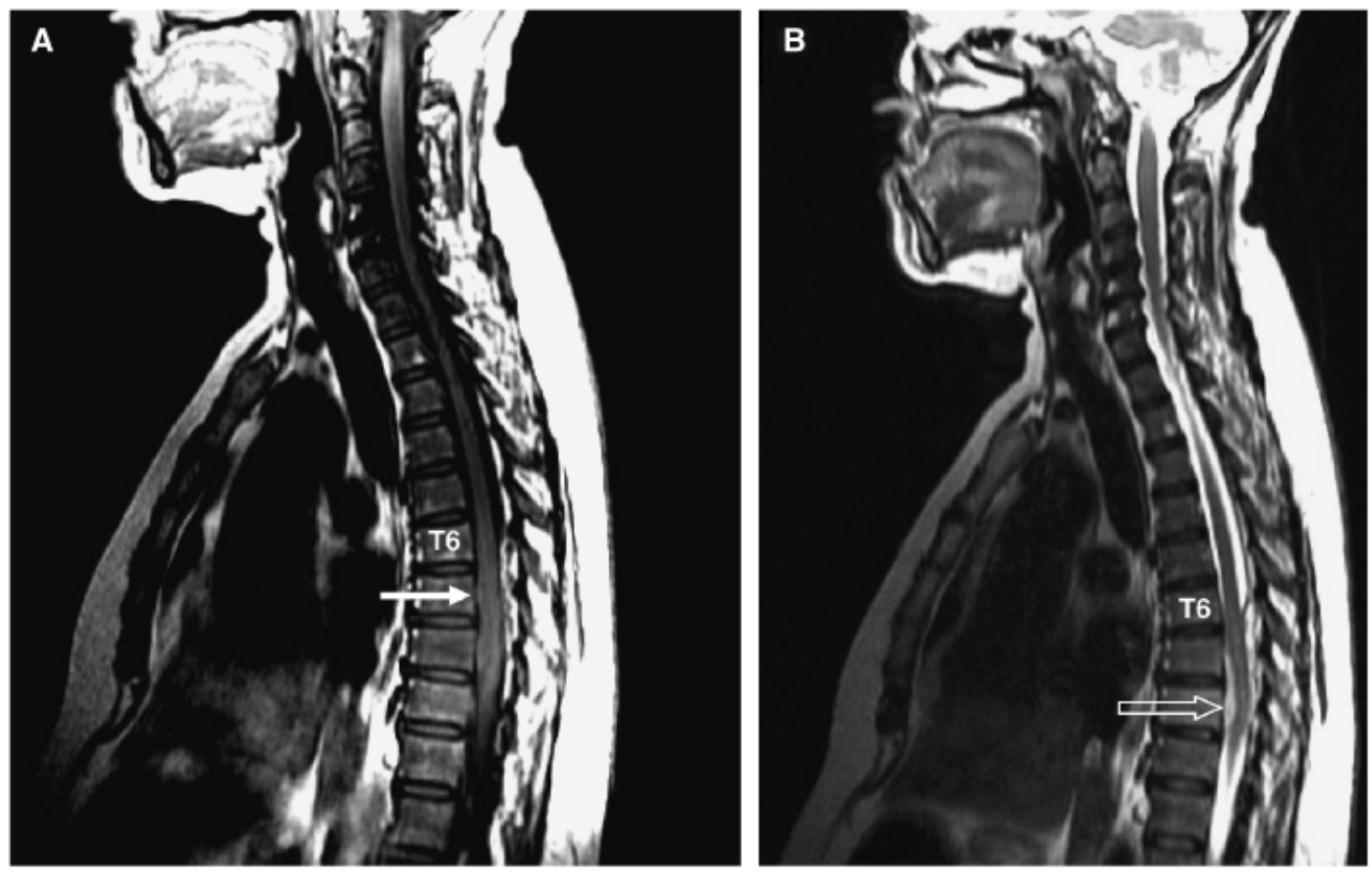

FIGURE 1. T1-weighted (A) sagittal MRI showing cord edema (solid white arrow) from 6th to 8th thoracic vertebral levels. T2-weighted (B) sagittal magnetic resonance imaging showing cord edema and collection of fluid (unfilled white arrow) at 7th thoracic vertebral level.

aureus, sensitive to methicillin, clindamycin, and sulfamethoxazole/trimethoprim, but resistant to penicillin (minimum inhibitory concentration $\geq 0.5 \mu \mathrm{g} / \mathrm{mL}$ ). She received a 9week course of antibiotics, initially with intravenous cloxacillin (9 g/day) for the first 3 weeks. Following the development of a rash attributed to cloxacillin, she was prescribed sulfamethox- azole/trimethoprim (cotrimoxazole 400/80 mg) 2 tablets twice daily, and clindamycin $900 \mathrm{mg} /$ day orally for an additional 5 weeks. Recovery was uneventful, and she was able to ambulate with minimal assistance within a week, power MRC grade $4 / 5$ (i.e., she was able to lift them against some, but not full, resistance). She was discharged to a rehabilitation facility,
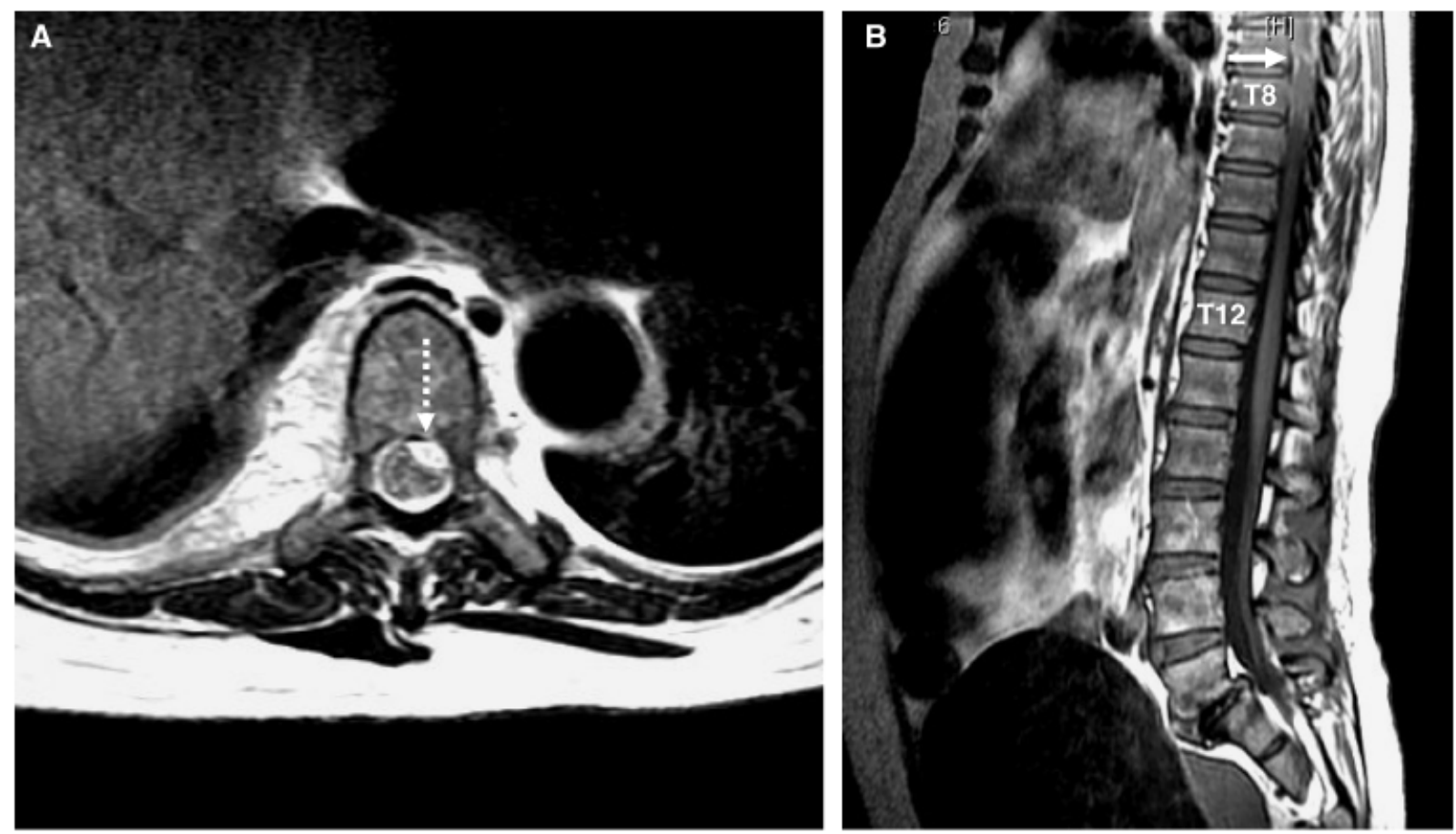

FIGURE 2. T2-weighted axial MRI at 8th thoracic vertebral level (A) showing left paracentral fluid collection (dotted white arrow), compressing on spinal cord. T2-weighted sagittal MRI (B) showing septated abscess (thick white arrow) at 8th thoracic vertebral level. 
and reviewed 1-month postoperatively, when she was able to ambulate independently, power MRC grade 5/5 (i.e., normal and able to overcome full resistance).

\section{DISCUSSION}

Epigastric pain usually arises from intraabdominal structures such as the upper gastrointestinal tract and the hepatobiliary system, as well as structures outside of the abdomen, e.g., heart and thoracic spine. ${ }^{7}$ Visceral pain is usually vague, poorly localized, and mid-line, while radicular pain is sharp, lancinating, ${ }^{8}$ and band-like when arising from the thoracic spine. The dermatomal representation of the 6 th to 8 th thoracic nerve roots in the epigastrium is consistent with the anatomic localization of the SEA and the development of the epigastric pain in our patient. Whitcomb et al. ${ }^{4}$ described a similar case, in whom repeated investigations and surgical procedures did not ameliorate abdominal pain until surgical decompression was performed.

Spinal epidural abscesses are rare and difficult to diagnose. They have a potentially devastating outcome, with mortality rates ranging from $4.6 \%$ to $31 \%,{ }^{9}$ especially in those with severe neurologic deficit at onset. ${ }^{9,10}$ However, outcome is markedly improved with early diagnosis and definitive surgical treatment. ${ }^{9}$ Spinal epidural abscesses may be seen in association with spinal osteomyelitis. ${ }^{9}$ They have been described in immunocompromised patients, e.g., diabetics, ${ }^{11}$ after invasive procedures such as lumbar puncture and epidural anesthesia have been performed. ${ }^{3}$ Spinal epidural abscess has also been described among intravenous drug users. In approximately one third of them, a source of infection cannot be identified. ${ }^{12}$ The susceptibility of poorly controlled diabetics to infection ${ }^{13}$ is thought to be secondary to impaired lymphocytic $^{14}$ and phagocytic function. ${ }^{15}$ As such, diabetics are prone to developing staphylococcal infections. ${ }^{16}$

Pereira and Lynch $^{9}$ identified blunt spinal trauma in 6 out of 24 cases of SEA. Patients with SEA commonly present with backache, radicular pain, weakness, sensory deficits, ${ }^{3}$ as well as vertebral tenderness, fever, and occult septicemia. ${ }^{11}$ Without having had a prior invasive procedure, it is difficult to picture how our patient developed a SEA. Possibly she had an occult infection, which spread to the spinal canal.

As in our patient, Staphylococcus aureus is most commonly cultured from SEA, although Gram-negative bacilli have more recently been found to play a more prominent role..$^{9,11}$ Spinal epidural abscesses most frequently involve the lumbar and thoracic spine, with the cervical spine only rarely being affected. ${ }^{11}$ The treatment is primarily surgical, with prompt decompressive laminectomy, and antibiotics normally continued for at least 4 to 6 weeks. ${ }^{9,11}$ Despite excellent results with surgical decompression, some authors advocate nonoperative management in patients with no evidence of cord compression, or when relevant cultures have identified the causative organism. ${ }^{17}$ Medical therapy may be preferable to surgical interven- tion when the patient is a poor surgical candidate, the extent of the abscess prohibits surgical decompression, when there are no neurologic deficits or when there has been complete paralysis for more than 3 days. ${ }^{18}$ Conversely, surgical decompression should be carried out when patients exhibit neurologic deficits. ${ }^{18}$

The diagnosis of SEA requires a high index of clinical suspicion. Even in the absence of "red flags" such as weakness, pain (especially band-like), paresthesias, urinary symptoms, spinal tenderness, and headaches, this neurologic emergency should be considered in any patient with severe localized back pain, raised inflammatory markers (erythrocyte sedimentation rate and $\mathrm{C}$-reactive protein), even in the absence of fever and neurologic deficit. ${ }^{2}$

\section{REFERENCES}

1. Darouiche RO, Hamill RJ, Greenberg SB, Weathers SW, Musher DM. Bacterial spinal epidural abscess: review of 43 cases and literature survey. Medicine (Baltimore). 1992;71:369-85.

2. Joshi SM, Hatfield RH, Martin J, Taylor W. Spinal epidural abscess: a diagnostic challenge. Br J Neurosurg. 2003;17:160-3.

3. Bremer AA, Darouiche RO. Spinal epidural abscess presenting as intraabdominal pathology: a case report and literature review. J Emerg Med. 2004;26:51-6.

4. Whitcomb DC, Martin SP, Schoen RE, Jho HD. Chronic abdominal pain caused by thoracic disc herniation. Am J Gastroenterol. 1995;90: 835-7.

5. Sellman MS, Mayer RF. Thoracoabdominal radiculopathy. South Med J. 1988;81:199-201.

6. Ditunno JF, Little JW, Tessler A, Burns AS. Spinal shock revisited: a four-phase model. Spinal Cord. 2004;42:383-95.

7. Sorkin LS, Wallace MS. Acute pain mechanisms. Surg Clin North Am. 1999;79:213-29.

8. Siddall PJ, Taylor DA, Cousins MJ. Classification of pain following spinal cord injury. Spinal Cord. 1997;35:69-75.

9. Pereira CE, Lynch JC. Spinal epidural abscess: an analysis of 24 cases. Surg Neurol. 2005;63(Suppl 1):S26-9.

10. Danner RL, Hartman BJ. Update on spinal epidural abscess: 35 cases and review of the literature. Rev Infect Dis. 1987;9:265-74.

11. Simpson J, Foinette KM, Lobo DN, Rowlands BJ. Spinal epidural abscess: adding insult to injury? Injury. 1999;30:504-8.

12. Curry WT Jr., Hoh BL, Amin-Hanjani S, Eskandar EN. Spinal epidural abscess: clinical presentation, management, and outcome. Surg Neurol. 2005;63:364-71.

13. Periti P, Mazzei T. Infections in immunocompromised patients. II. Established therapy and its limitations. Clin Ther. 1985;8:100-17.

14. Plouffe JF, Silva J Jr., Fekety R, Allen JL. Cell-mediated immunity in diabetes mellitus. Infect Immun. 1978;21:425-9.

15. Marhoffer W, Stein M, Schleinkofer L, Federlin K. Evidence of ex vivo and in vitro impaired neutrophil oxidative burst and phagocytic capacity in type 1 diabetes mellitus. Diabetes Res Clin Pract. 1993;19:183-8.

16. Weems JJ Jr. The many faces of Staphylococcus aureus infection. Recognizing and managing its life-threatening manifestations. Postgrad Med. 2001;110:24-36.

17. Mampalam TJ, Rosegay H, Andrews BT, Rosenblum ML, Pitts LH. Nonoperative treatment of spinal epidural infections. J Neurosurg. 1989; 71:208-10.

18. Tang HJ, Lin HJ, Liu YC, Li CM. Spinal epidural abscess-experience with 46 patients and evaluation of prognostic factors. J Infect. 2002;45: 76-81. 